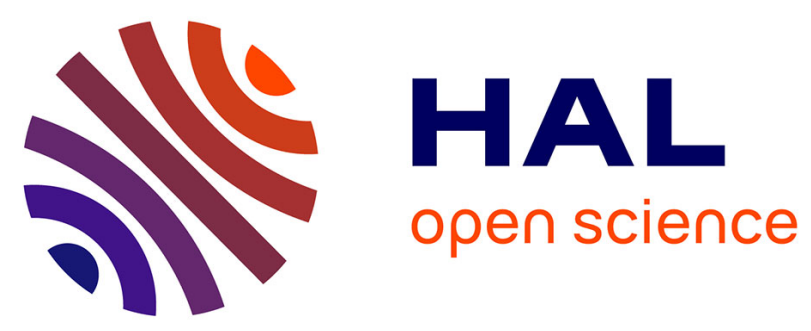

\title{
Biomimetic neural network for modifying biological dynamics during hybrid experiments
}

Matthieu Ambroise, Stefano Buccelli, Filippo Grassia, Antoine Pirog, Yannick Bornat, Michela Chiappalone, Timothée Levi

\section{- To cite this version:}

Matthieu Ambroise, Stefano Buccelli, Filippo Grassia, Antoine Pirog, Yannick Bornat, et al.. Biomimetic neural network for modifying biological dynamics during hybrid experiments. 22th International Symposium on Artificial Life and Robotics, Jan 2017, Beppu, Japan. hal-01567497

\section{HAL Id: hal-01567497 \\ https://hal.science/hal-01567497}

Submitted on 27 Jul 2017

HAL is a multi-disciplinary open access archive for the deposit and dissemination of scientific research documents, whether they are published or not. The documents may come from teaching and research institutions in France or abroad, or from public or private research centers.
L'archive ouverte pluridisciplinaire HAL, est destinée au dépôt et à la diffusion de documents scientifiques de niveau recherche, publiés ou non, émanant des établissements d'enseignement et de recherche français ou étrangers, des laboratoires publics ou privés. 


\title{
Biomimetic neural network for modifying biological dynamics during hybrid experiments
}

\author{
Matthieu Ambroise ${ }^{1}$, Stefano Buccelli ${ }^{2}$, Filippo Grassia ${ }^{3}$, Antoine Pirog ${ }^{1}$, Yannick Bornat $^{1}$, Michela Chiappalone ${ }^{2}$, \\ Timothée Levi ${ }^{1}$ \\ ${ }^{1}$ IMS Lab., University of Bordeaux, France \\ ${ }^{2}$ Department of Neuroscience and Brain Technologies, Istituto Italiano di Tecnologia, Genova, Italy \\ ${ }^{3}$ LTI Lab., University of Picardie Jules Verne, France \\ (Tel: 33-5-4000-3118, Fax: 33-5-5637-1545) \\ 1timothee.levi@u-bordeaux.fr
}

\begin{abstract}
Electrical stimulation of nerve tissue and recording of neural electrical activity are the basis of emerging prostheses and treatments for many neurological disorders. Here we present closed-loop biohybrid experiment using in vitro Biological Neuronal Network (BNN) with an Artificial Neural Network (ANN) implemented in a neuromorphic board. We adopted a neuromorphic board which is able to perform real-time event detection and trigger an electrical stimulation of the BNN. This system embeds an ANN, based on Izhikevich neurons which can be put in uni- and bi-directional communication with the BNN. The ANN used in the following experiments was made up of 20 excitatory neurons with inhibition synapse and with synaptic plasticity to design Central Pattern Generator (CPG). Open-loop and closed-loop hybrid experiments shows that the biological dynamics can be modified. This work can be seen as the first step towards the realization of an innovative neuroprosthesis.
\end{abstract}

Keywords: neuromorphic engineering, biological neural network, artificial neural network, CPG, bio-hybrid experiments

\section{INTRODUCTION}

Neurological disorders affect millions of people around the world which influence their cognitive and/or motor capabilities. Evolutions of the technology and the increasing number of engineer have changed the perception of the medical treatment. Besides chemical processes of healing, devices are developed in order to improve the life quality. The realization of a prosthesis must consider the biological activity of the cells and the connection between machine and biological cells. The realization of such neuroprostheses [14] implies that we know how to interact with neuronal cell assemblies, taking into account the intrinsic spontaneous activity of neuronal networks and understanding how to drive them into a desired state or to produce a specific behavior. The long-term goal of replacing damaged brain areas with artificial devices also requires the development of neuronal network models. They will fit with the recorded electrophysiological patterns and will produce in their turn the correct stimulation patterns for the brain so as to recover the desired function.

The hardware set-up that will be used to interface the biological component is a biomimetic Spiking Neural Network (SNN) system [5-8]. This SNN implements biologically realistic neural network models, spanning from the electrophysiological properties of one single neuron up to network plasticity rules. SNN is one solution for new generation of neuroprosthesis and to perform hybrid experiments between biological cells and artificial neurons.

Here, we propose a biomimetic neural network implemented in FPGA connected in a bi-directional communication with living neurons cultured in MicroElectrode Array (MEA) thanks to a neuromorphic board which detect neuronal activity and send pattern stimulation to the culture. We performed experiments in open-loop (Biological $\Rightarrow$ Artificial) and in closed-loop (Biological $\Leftrightarrow$ Artificial). This part constitutes one main point of the FET7 European project Brainbow.

\section{CLOSED-LOOP PLATFORM}

\subsection{Neuron culture and recording/stimulation system}

The biological element used in this study is constituted by dissociated cortical rat neurons plated over MicroElectrode Arrays (MEAs). Cultures were prepared as described in [9].

'In vitro' cultured neuronal networks coupled to microelectrode arrays (MEAs) constitute a valuable experimental model for studying changes in the neuronal dynamics at different stages of development. After a few days in culture, neurons start to connect each other with functionally active synapses, forming a random network and displaying spontaneous electrophysiological activity. We are using these neuron cultures on MEAs for closed-loop experiments. 
Cortical cultures were plated on 60 planar microelectrodes (MultiChannel Systems, Reutlingen, Germany) arranged over an $8 \times 8$ square grid (with the four corner electrodes not present). After $1200 \times$ amplification (MEA 1060, MCS, Reutlingen, Germany), signals were sampled at $10 \mathrm{kHz}$ using the MultiChannel System data acquisition card controlled by the commercial software MC_Rack (MCS, Reutlingen, Germany). The stimulation part was carried out by employing the MultiChannel System stimulator STG4002

\subsection{Multimed Board}

Multimed is a multi-application acquisition and signal processing board. It is specifically designed for the real-time analysis of multichannel biological data in closed-loop configurations. It features $10 \mathrm{kHz}, 16$-bits acquisition on 64 channels, application-specific processing chains with submillisecond processing latency and decision-making, live display, computer control interfaces, and recording capabilities. The processing chains typically include linear band-pass filters, wavelet filters, Action Potential (AP) and Local Field Potential (LFP) detection, single and network burst detection, digital stimulus artifact blanking and flexible event routing to define complex feedback rules. All processing functions can be accessed and configured via a computer interface. In order to enhance configurability and lower the processing latency, the described architecture is implemented on an FPGA, as are the SD card recording, VGA display, and serial control interfaces.

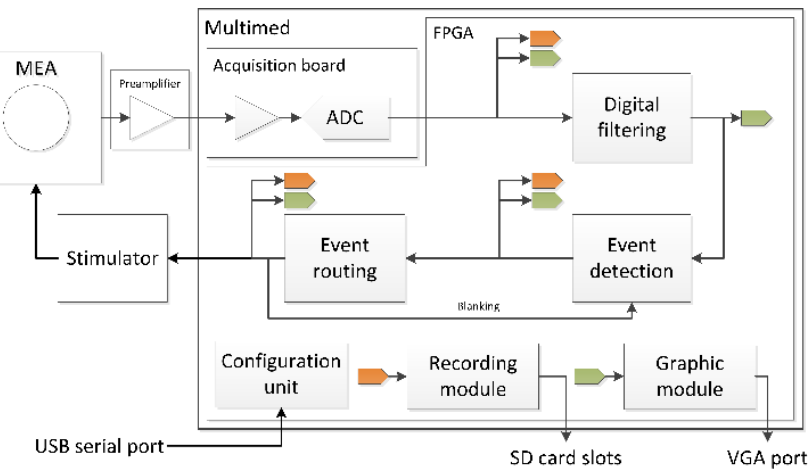

Fig. 1. Description of the Multimed system. The data accessible for recording and display are marked by the orange and green ports.

The MEA, external preamplifier, and Multimed input ports are MCS-compatible. The Multimed stimulator output is a TTL line. The acquisition board includes a preamplifier and analog-to-digital converters. The processing chain includes digital filtering (band-pass and wavelet filtering), event detection (AP, LFP, and burst detection), and event routing.
On the FPGA of the Multimed board, we implement Spiking Neural Network (SNN). This part is described in the next section.

\section{Digital Spiking Neural Network (SNN)}

\subsection{Choice of Technology}

Various software solutions are currently available for simulating neuron models. Less conventional than softwarebased systems, hardware based solutions are also provided which generally combine digital and analog forms of computation. Analog implementations are fast and efficient; they are inflexible, sensitive to variations and require a long development time.

As in many other fields of microelectronics, a mixed implementation offers both the advantages and disadvantages of both solutions: analog circuits have a higher integration density, and digital platforms have better programmability.

Recently, as a midpoint in the design space, FPGAs have been used to build spiking neuronal networks. Digital FPGA implementations offer a significant speedup over software designs, as well as size, weight, and power efficiency. Compared to analog VLSI, digital FPGAs designs are stable and flexible in design alterations. Previous works have already implemented neuronal networks on FPGA [10-14]. However, those designs have been realized for computation purposes without taking into account biological real time. We decided to choose FPGA implementation.

\subsection{Choice of neuron model and synapse}

In the human brain, types of neurons are radically different. The neuronal model is a mathematical description of the electrophysiological properties of neuronal cells, or neurons. It tries to accurately describe and predict their biological processes.

In order to provide Spiking Neural Network (SNN), the first step is the choice of the neuromimetic mathematical model. Indeed, the mathematical model, based on differential equation, can reproduce a behavior more or less close to biological cell. In order to choose our model, we are looking two criteria: the variety of firing rate dynamics that can be reproduced and the number of equation used for computation.

Regarding those criteria, we can compare all models like the Leaky Integrate and Fire (LIF) [15], the Hodgkin-Huxley model (HH) [16], or the Izhikevich model (IZH) [17].

$\mathrm{HH}$ model requires a large number of parameters and the LIF model is too simple for reproducing biomimetic neural network like this CPG [18]. 
The Izhikevich model (IZH) represents a good trade-off, as it is based on two equations and is capable of reproducing many different families of neurons by changing four parameters. Furthermore, according to [17], this model is resource-frugal, a key advantage when the aim is to design a large Central Pattern Generator CPG [19] network embedded in the same board. The IZH model depends on four parameters, a, b, c and d which make it possible to reproduce the spiking and bursting behavior of specific types of cortical neurons. From a mathematical standpoint, the model is described by a two-dimensional system of ordinary differential equations:

$$
\begin{gathered}
\frac{d v}{d t}=0.04 v^{2}+5 v+140-u+I_{I z h} \\
\frac{d u}{d t}=a(b v-u)
\end{gathered}
$$

With the after-spike resetting conditions:

$$
\text { if } v \geq 30 m V \Rightarrow\left\{\begin{array}{l}
v \leftarrow c \\
u \leftarrow u+d
\end{array}\right.
$$

To validate the neuron implementation, we compared the firing curves of fast spiking (FS) and regular spiking (RS) neuron family of the artificial network to the model ones. The RS neurons are used for the CPG neural network.

A neural network is defined by a group of neurons and synapses. As we chose the neuron model, we have to choose carefully the synapse model. This model should be biomimetic and using few resources. A synapse connects a presynaptic neuron to a postsynaptic one. In case of excitatory (inhibitory) synapse, a positive contribution $I_{\text {exc }}$ (negative contribution $\mathrm{I}_{\text {inh }}$ ) is added to the postsynaptic neuron when the presynaptic neuron spikes. Those both kinds of synapse are well known in biology: AMPA and $\mathrm{GABA}_{\mathrm{a}}$ for excitatory and inhibitory synapses respectively. The synaptic current rising lasts $1 \mathrm{~ms}$ that is the computation time step. Then it will exponentially decrease until zero. The constant time of excitatory decay $\left(\tau_{\text {exc }}\right)$ and of inhibitory decay $\left(\tau_{\text {inh }}\right)$ are different, i.e. are equal to $3 \mathrm{~ms}$ and $10 \mathrm{~ms}$ respectively in the neuroscientist synapse model [20]. Furthermore, we add the short-term plasticity described by Izhikevich [17] that is different from Spike-Timing Dependent Plasticity (STDP) and that manages the depression or the facilitation of the synaptic strength.

\subsection{Architecture of the Spiking Neural Network}

The architecture of the network implementation is based on RAM blocks (that store all parameters needed to define a network), two computation cores (one used for neurons and the other one for synapses), a block to manage the state machine and the addresses and a block "Communication RS232" (that will allow us to configure/change parameters of our system). It is freely configurable from an independentneuron configuration to different neural network configurations with different options like the synaptic plasticity, the synaptic noise and the axonal delay. Furthermore, this implementation used a few part of resources and it uses pipeline implementation.

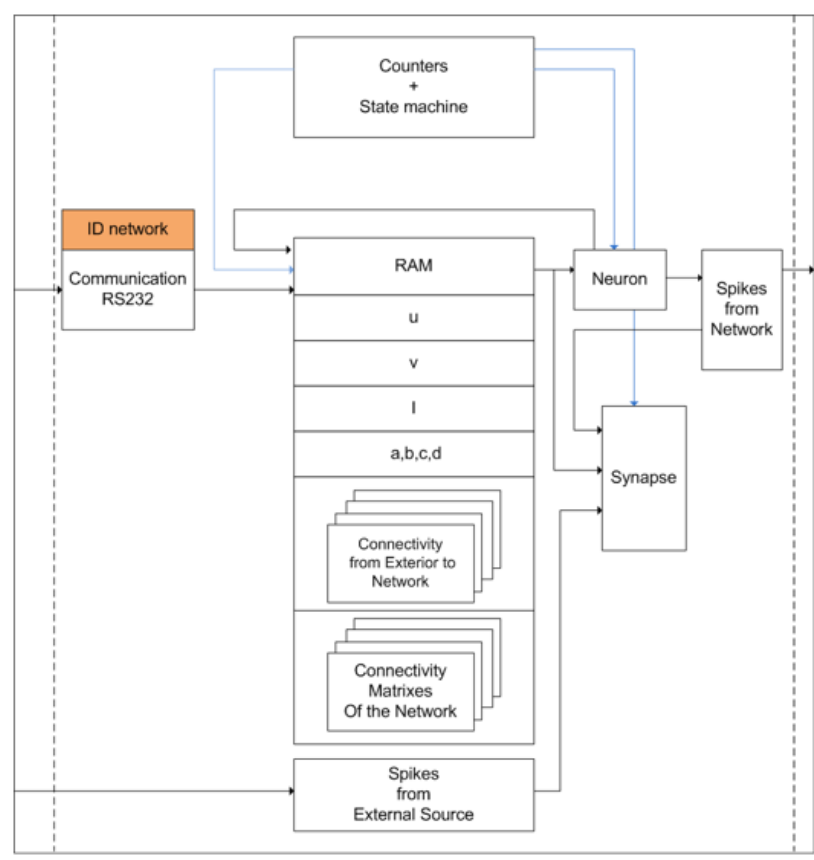

Fig. 2. Description of the SNN architecture

Our architecture can be also freely configurable from an independent-neuron configuration to all-to-all configuration or a mixed with small networks and independent neurons.

\section{BIOHYBRID EXPERIMENTS}

\subsection{Biomimetic Central Pattern Generator (CPG)}

From [13], [21], we can configure biomimetic CPGs for biohybrid experiments. CPGs are neural networks capable of producing rhythmic patterned outputs without rhythmic sensory or central input. We implement two CPGs using 20 Izhikevich neurons (16 for CPGs and 4 for stimulation triggers) with 24 inhibitory synapses. Synaptic plasticity is also included in this SNN.

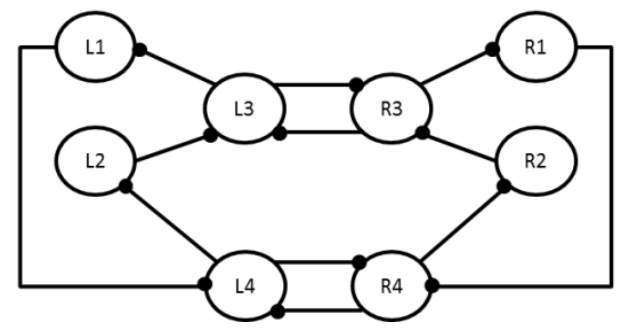

Fig. 3. A diagram of the neural network describing one CPG including two elemental oscillators, L3/R3 and L4/R4, 
and two pairs of coordination neurons, L1/R1 and L2/R2. The synapses are inhibitory. The network contains synaptic plasticity.

Figure 4 describes the activity of 2 CPGs, one at a $2.6 \mathrm{~s}$ period and one at a $6.8 \mathrm{~s}$ period. When the CPG burst begins, a stimulation is sent to the BNN. For bio-hybrid experiments, we can use two kinds of pattern stimulations (CPG1 or CPG2).

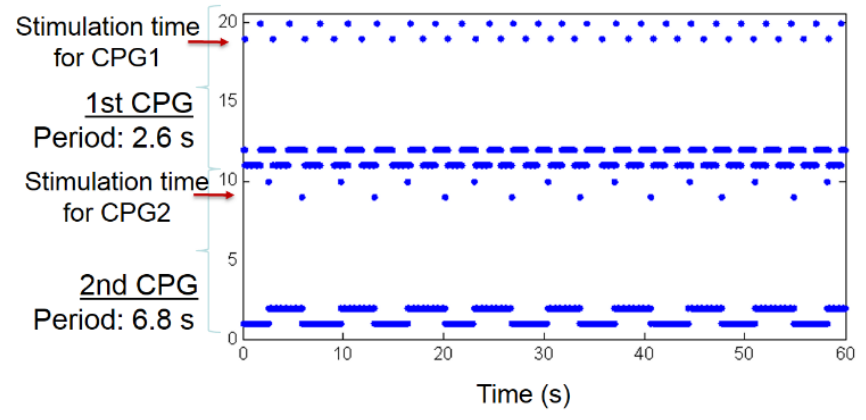

Fig. 4. Description of the two CPGs activity and the two pattern stimulation time.

\subsection{Open-Loop experiments}

The first bio-hybrid experiment is to use the BNN as an inhibition stimulator to the ANN composed of two CPGs. Figure 5 describes the BNN behavior (electrode 1 to 60) and its spontaneous activity. When a network burst is detected by Multimed board, an inhibition stimulation is sent to the two CPGs. We notice that CPG activities (neuron 61 to 80 ) are stopped during few seconds after each stimulation.

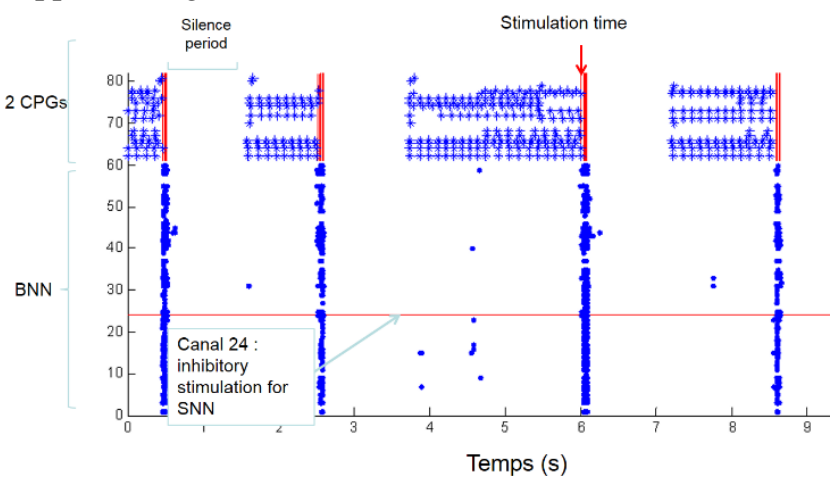

Fig. 5. Open-loop experiments. The BNN sends inhibition stimulation to the CPG when a network burst occurs.

\subsection{Closed-loop experiments}

The closed-loop is much complex. From BNN to SNN, the procedure is the same than for the open-loop experiments. To close the loop, we perform an excitatory stimulation to the BNN when the CPG begin a burst activity.

The canal 24 that corresponds to BNN part, sends an inhibition to SNN part. The artificial neuron 79 sends an excitatory stimulation to BNN when it spikes. The neuron 79 in Figure 5 and 6 corresponds to neuron 19 in Figure 4.

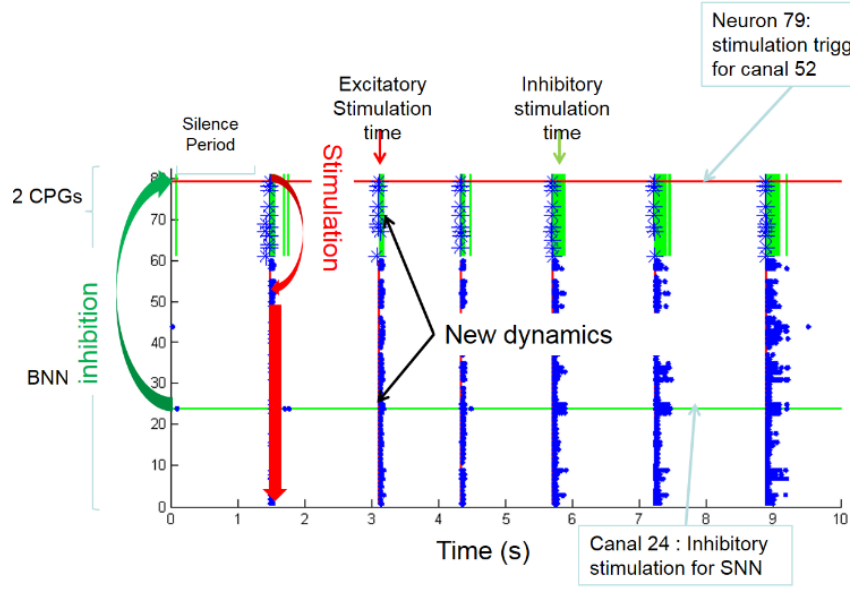

Fig. 6. Closed-loop experiments. The BNN sends inhibition stimulation to the CPG when a network burst occurs. The CPG sends excitatory stimulation to BNN when a CPG burst begin.

Figure 6 describes important behaviors. Indeed, we notice that the BNN and SNN merge to one single activity. And this activity is different from the initial ones (for BNN and for SNN). This activity is periodic. It implies that depending the stimulation strength (for inhibition and for excitation), we can modify this periodic activity and used it for different applications like bio-hybrid robotics. The biological dynamic is then modified and can be controlled depending the SNN configuration and the stimulation intensity.

\section{CONCLUSION}

We presented, in this paper, bio-hybrid experiments using neuron culture on MEA, a neuromorphic board for recording and stimulation and SNN. Open-loop and closed-loop experiments are performed using CPG as artificial neural network. We notice that the dynamic of the BNN is modified and merge with the SNN into a new periodic dynamic.

The final goal of the studies is to build a test-bed for the study and the development of a new generation of neuroprostheses capable to restore the lost communication between neuronal circuitries. The presented experimental and computational platforms represent a starting point for restoring a functional closed-loop communication in a neuronal network with lesioned circuitries.

\section{ACKNOWLEDGMENTS}

The research leading to these results has received funding from the European Union's Seventh Framework Programme (ICTFET FP7/2007-2013, FET Young Explorers scheme) 
under grant agreement n. 284772 BRAIN BOW (www.brainbowproject.eu).

\section{REFERENCES}

[1] Nicolelis, M. A.L., and Lebedev, M. A. (2009), Principles of neural ensemble physiology underlying the operation of brain-machine interfaces, Nature Reviews Neuroscience, 10, 530-540

[2] Hochberg, L.R., Serruya, M.D., Friehs, G.M., Mukand, J.A., Saleh, M., Caplan, A.H., Branner, A., Chen, D., Penn, R.D., and Donoghue, J.P. (2006), Neuronal ensemble control of prosthetic devices by a human with tetraplegia, Nature, 442, 164-171

[3] Hochberg, L.R., Bacher, D., Jarosiewicz, B., Masse, N.Y., Simeral, J.D., Vogel, J., Haddadin, S., Liu, J., Cash, S.S., Van Der Smagt, P., and Donoghue, J.P. (2012), Reach and grasp by people with tetraplegia using a neurally controlled robotic arm, Nature Methods, 485, 372-375

[4] Bonifazi P, Difato F, Massobrio P, Breschi GL, Pasquale V, Levi T, Goldin M, Bornat Y, Tedesco M, Bisio M, Kanner S, Galron R, Tessadori J, Taverna S and Chiappalone M (2013), In vitro large-scale experimental and theoretical studies for the realization of bi-directional brainprostheses. Frontiers in Neural Circuits, 7:40

[5] Levi T, Lewis N, Tomas J, Saighi S, Renaud S, Bornat Y, Alvado L (2008), Neuromimetic Integrated Circuits, VLSI Circuits for Biomedical Applications, Iniewski Editor

[6] Mahowald M., R. Douglas R. (1991), A Silicon neuron, Nature, 1991: 515-518

[7] Indiveri G. et al. (2001), Neuromorphic silicon neuron circuits, Frontiers in Neuroscience, 5:73

[8] Levi T, Lewis N, Tomas J, Fouillat P (2008), IP-based methodology for analog design flow: application on neuromorphic engineering, NEWCAS-TAISA Conference, 343-346

[9] Frega M, Pasquale V, Tedesco M, Marcoli M, Contestabile A, Nanni M, Bonzano L, Maura G, and Chiappalone M (2012), Cortical cultures coupled to microelectrode arrays: a novel approach to perform in vitro excitotoxicity testing, Neurotoxicol Teratol, 34:116-127

[10] Cassidy, A., Andreou, A.G (2008), Dynamical digital silicon neurons, IEEE Biomedical Circuits and Systems Conference, pp.289-292, 20-22

[11] Cassidy, A., Andreou, A.G., Georgiou, J. (2011), Design of a one million neuron single FPGA neuromorphic system for real-time multimodal scene analysis, 45th Annual Conference CISS, 1-6

[12] Basham, E.J, Parent D.W (2012), Compact digital implementation of a quadratic integrate-and-fire neuron, 34th Annual Conference of the IEEE EMBS, 3543-8

[13] Ambroise M, Levi T, Joucla S, Yvert B, Saighi S. (2013), Real-time biomimetic Central Pattern Generators into FPGA for hybrid experiments, Frontiers in Neuroscience, 7: 215

[14] Grassia F., Levi T., Kohno T., Saighi S. (2014), Silicon neuron: digital hardware implementation of the quartic model, Journal of Artificial Life and Robotics, 19:215-219
[15] Indiveri G (2007), Synaptic plasticity and spikebased computation in VLSI networks of integrate-and-fire neurons. Neural Information Processing, Letters and Reviews 11:135-146

[16] Izhikevich, E.M. (2003), Simple model of spiking neurons, IEEE Transactions on Neural Networks, vol.14, no.6, 1569-1572

[17] Hodgkin AL, Huxley AF (1952), A quantitative description of membrane current and its applications to conduction and excitation in nerve, The Journal of Physiology, 117:500-544

[18] Hill AA., Lu J., Masino MA. , Olsen OH, Calabrese RL. (2001). A model of a segmental oscillator in the leech heartbeat neuronal network, J Comput Neurosci., 10, 281 302.

[19] Marder E., Bucher D. (2001). Central pattern generators and the control of rhythmic movements. Current Biology 2001,11, 986-996.

[20] Destexhe,A., Mainen, Z.F. and Sejnowski, T. J. (1994). An efficient method for computing synaptic conductances based on a kinetic model of receptor binding, Neural Computation, 6, 14-18

[21] Joucla S, Ambroise M, Levi T, Lafon T, Chauvet P, Saïghi S, Bornat Y, Lewis N, Renaud S and Yvert B (2016), Generation of Locomotor-Like Activity in the Isolated Rat Spinal Cord Using Intraspinal Electrical Microstimulation Driven by a Digital Neuromorphic CPG. Frontiers in Neurosciences, 10:67 